\title{
PARAMETRIC RESONANCE AND RADIATIVE DECAY OF DISPERSION-MANAGED SOLITONS*
}

\author{
DMITRY E. PELINOVSKY ${ }^{\dagger}$ AND JIANKE YANG ${ }^{\ddagger}$
}

\begin{abstract}
We study propagation of dispersion-managed solitons in optical fibers which are modeled by the nonlinear Schrödinger equation with a periodic dispersion coefficient. When the dispersion variations are weak compared to the average dispersion, we develop perturbation series expansions and construct asymptotic solutions at the first and second orders of approximation. Due to a parametric resonance between the dispersion map and the dispersion-managed soliton, the soliton generates continuous-wave radiation leading to its radiative decay. The nonlinear Fermi golden rule for radiative decay of dispersion-managed solitons is derived from the solvability condition for the perturbation series expansions. Analytical results are compared to direct numerical simulations, and good agreement is obtained.
\end{abstract}

Key words. dispersion management, optical solitons, perturbation series, parametric resonance, radiative decay, Fermi golden rule

AMS subject classifications. 35Q55, 78M30, 78M35

DOI. $10.1137 /$ S0036139903422358

1. Introduction. This paper addresses the dispersion-periodic nonlinear Schrödinger (NLS) equation,

$$
i \frac{\partial u}{\partial z}+\frac{m}{2 \epsilon} D_{\epsilon}(z) \frac{\partial^{2} u}{\partial t^{2}}+\frac{1}{2} D_{0} \frac{\partial^{2} u}{\partial t^{2}}+|u|^{2} u=0,
$$

which models optical pulse propagation in dispersion-managed communication systems. Here $u \in \mathbb{C}$ is the wave envelope of the electromagnetic field, $z(\geq 0)$ is the distance along the optical fiber, $t \in \mathbb{R}$ is the retarded time of the optical pulse, $D_{0}$ is the average dispersion, $D_{\epsilon}(z)$ is an $\epsilon$-periodic mean-zero dispersion map, and $m$ is the strength of the map variations. Lump amplification and losses are not included in the model (1.1) for the sake of simplicity.

Special solutions of the dispersion-periodic NLS equation (1.1) are called dispersion-managed (DM) solitons. They have been the subject of growing interest in recent literature $[1,2,3]$. DM solitons are periodic solutions of (1.1) in the form

$$
u(z, t)=\Phi(z, t) e^{i \mu z},
$$

where $\Phi(z+\epsilon, t)=\Phi(z, t)$ and $\mu \in \mathbb{R}$. Existence of periodic solutions of (1.1) is studied with the normal-form transformations in the limit $\epsilon \rightarrow 0$ [4]. The normalform transformations average the fast periodic variations of $\frac{1}{\epsilon} D_{\epsilon}(z)$ and reduce the dispersion-periodic NLS equation (1.1) to an integral NLS equation [5, 6]. Bound states of the integral NLS equation exist in the case of $D_{0}>0[7]$ and in the case

*Received by the editors February 6, 2003; accepted for publication (in revised form) November 1, 2003; published electronically May 20, 2004.

http://www.siam.org/journals/siap/64-4/42235.html

${ }^{\dagger}$ Department of Mathematics, McMaster University, 1280 Main Street West, Hamilton, Ontario, Canada, L8S 4K1 (dmpeli@math.mcmaster.ca). The work of this author was supported by NSERC grant 5-36694.

${ }^{\ddagger}$ Department of Mathematics, University of Vermont, Burlington, VT 05401 (jyang@emba.uvm. edu). The work of this author was supported by NSF grant DMS-9971712 and by a NASA EPSCoR minigrant. 
$D_{0}=0[8]$. Numerical results indicate nonexistence of bound states of the integral NLS equation in the case $D_{0}<0$ [9].

In what follows we consider the case $D_{0}>0$ only. Early papers by Nijhof et al. [11] reported numerically the existence of "exactly" periodic bound states in the dispersion-periodic NLS equation (1.1), which do not radiate any energy. Later, more careful numerics [3] showed that such bound states actually had nonvanishing radiation tails. Recent results of Yang and Kath [10] showed that exactly-periodic DM solitons do not exist in the dispersion-periodic NLS equation (1.1) because resonances in the perturbation series generate nonvanishing radiation tails. These tails can be extremely small in certain parameter regimes [10], but they do not vanish when $D_{0}>0$.

Radiation tails of DM solitons occur due to parametric resonance between the DM soliton and the periodic variation of the dispersion. This parametric resonance drains energy out of the DM soliton and leads to its radiative damping. Parametric resonances can be predicted by viewing the periodic term of (1.1) as an external forcing term:

$$
i \frac{\partial u}{\partial z}+\frac{1}{2} D_{0} \frac{\partial^{2} u}{\partial t^{2}}+|u|^{2} u=-\frac{m}{2 \epsilon} D_{\epsilon}(z) \frac{\partial^{2} u}{\partial t^{2}} .
$$

We expand $D_{\epsilon}(z)$ into a Fourier series,

$$
D_{\epsilon}(z)=\sum_{n=-\infty}^{\infty} d_{n} e^{\frac{2 \pi i n z}{\epsilon}}, \quad d_{0}=0, \quad d_{-n}=\bar{d}_{n},
$$

where $\bar{d}_{n}$ is the complex conjugate of $d_{n}$. When the nonlinear term in (1.3) is neglected and the averaged DM soliton $u(z, t)=\Phi(t) e^{i \mu z}$ is substituted into the right-hand side of (1.3), we find a solution of the linear inhomogeneous problem in the form of the Fourier series in $z$,

$$
u(z, t)=\left(\sum_{n=-\infty}^{\infty} u_{n}(t) e^{\frac{2 \pi i n z}{\epsilon}}\right) e^{i \mu z} .
$$

The correction terms $u_{n}(t)$ take the form of Fourier integrals in $t$,

$$
u_{n}(t)=-\frac{m d_{n}}{4 \pi \epsilon} \int_{-\infty}^{\infty} \frac{\omega^{2} \hat{\Phi}(\omega) e^{i \omega t} d \omega}{\frac{1}{2} D_{0} \omega^{2}+\frac{2 \pi n}{\epsilon}+\mu},
$$

where $\hat{\Phi}(\omega)$ is the Fourier transform of $\Phi(t)$. The inhomogeneous solution has resonant denominators at

$$
\omega^{2}=\omega_{n}^{2}=-\frac{2}{D_{0}}\left(\mu+\frac{2 \pi n}{\epsilon}\right)>0 .
$$

Resonances are absent if $D_{0}=0$ and $\mu \neq-2 \pi n / \epsilon$ for any integer $n$. This is the only case when DM soliton solutions (1.2) may exist in the dispersion-periodic NLS equation (1.1). In this case, the asymptotic representation of $\Phi(z, t)$ in (1.2) was found recently in [12] in the limit $\epsilon=\mathrm{O}(m) \gg 1$ with the use of the inverse scattering transform methods.

If $D_{0}>0$, sufficiently large negative terms of the Fourier series (1.5)-(1.6) are in resonance (1.7) for $n \leq-N_{\mu}$, where $N_{\mu}=\left[\frac{\epsilon \mu}{2 \pi}\right]$ is the integer ceiling of $\frac{\epsilon \mu}{2 \pi}>0$. The periodic variations of the dispersion map $D_{\epsilon}(z)$ lead to a coupling of a bound state 
and linear waves of the averaged dispersion map and to the energy transfer from the bound state to radiative waves. As a result, the pulse solution has resonant peaks in the spectrum $\hat{u}(z, \omega)$ at $\omega= \pm \omega_{n}$, and nonzero values of $u(z, t)$ in the far-field $|t| \gg 1$, as reported numerically in $[3,10]$.

Radiation damping of solitons in the presence of a weak sinusoidal dispersion variation was considered analytically in [13]. The radiative wave amplitudes and decay rates of solitons were computed by means of the soliton perturbation theory for the standard NLS equation. Dynamics of DM solitons was studied in $[14,15,16]$ by variational and numerical methods. Recently, analytical and numerical studies of the same problem were undertaken in [10] by asymptotic beyond-all-orders methods in the limit $\epsilon=\mathrm{O}(m) \ll 1$. Radiation-tail amplitudes and decay rates of DM solitons were found to be exponentially small in this limit. It was also shown in [10] that radiation-tail amplitudes drop to near-zero values in certain windows on the $m$-axis.

We study here nonlinear parametric resonance of DM solitons for average-anomalous dispersion $\left(D_{0}>0\right)$ in the limit $m \ll 1$, while we keep $\epsilon=\mathrm{O}(1)$. This is a different limit from the one studied in [10]. In this limit, the DM soliton decays much faster because radiation-tail amplitudes are only algebraically small in terms of $\mathrm{O}(m)$. The new feature of our analysis is that the periodic dispersion map $D_{\epsilon}(z)$ is allowed to be arbitrary in (1.4) as compared to a single sine function in [13]. Thus, our dispersion maps include the piecewise-constant dispersion map which is widely used in fiber communication systems.

Our analysis starts with the standard NLS equation (1.3) for $m=0$, such that the right-hand side of (1.3) is treated as a small perturbation. The first-order perturbation theory describes generation of linear waves due to parametric resonances (1.7), and the second-order perturbation theory leads to the decay rate of DM solitons. Methods of our analysis are similar to the soliton perturbation theory in [13], but our calculations are more systematic. We find that the DM soliton decays according to a nonlinear Fermi golden rule, which generalizes the Fermi golden rule for radiative decay of bound states in the linear Schrödinger equation with a time-periodic potential. Rigorous analysis of decay rates in the linear Schrödinger equation was recently considered in $[17,18]$, where the bound states were supported by a time-dependent periodic potential in [17] and by a time-independent potential in [18].

This paper is structured as follows. Section 2 contains perturbation series expansions and derivations of the Fermi golden rule for DM solitons. Section 3 is devoted to analytical approximations of radiative decay of DM solitons. Section 4 describes a comparison between the analytical and numerical results. Section 5 concludes the paper. Appendices A and B describe technical details of the first-order solution in the perturbation series expansions.

2. Perturbation series expansions. We start with the dispersion-periodic NLS equation in the form (1.3), where $\epsilon$ is finite and $m$ is small. If $D_{0}>0$, we employ the following rescaling of variables:

$$
z=\epsilon \hat{z}, \quad u=\frac{\hat{u}}{\sqrt{\epsilon}}, \quad t=\sqrt{\epsilon D_{0}} \hat{t}, \quad m=\epsilon D_{0} \hat{m} .
$$

When the hats are dropped, (1.3) becomes

$$
i u_{z}+\frac{1}{2} u_{t t}+|u|^{2} u=-\frac{m}{2} D_{1}(z) u_{t t},
$$

where the dispersion map $D_{1}(z)$ has unit period. In other words, we have normalized $\epsilon$ and $D_{0}$ in (1.3) so that $\epsilon=1$ and $D_{0}=1$. 
When $m=0$, the standard NLS equation (2.2) has a bound state:

$$
u(z, t)=\Phi(t ; \mu) e^{i \mu z},
$$

where $\mu>0$ and $\Phi(t ; \mu)=\sqrt{2 \mu} \operatorname{sech}(\sqrt{2 \mu} t)$. When $m \neq 0$, the NLS soliton (2.3) would generate radiative tails and decay accordingly. Parameter $\mu$ of the NLS soliton (2.3) changes in $z$, such that the $z$-dependence of $\mu(z)$ serves as a condition for Poincaré continuation of the perturbation series for $u(z, t)$ in powers of $m$. The Fermi golden rule of radiative decay of NLS solitons follows from the dynamical equation for $\mu=\mu(z)$. In order to formalize this qualitative picture, we employ the transformation

$$
u(z, t)=U(z, t ; \mu(z)) e^{i \int_{0}^{z} \mu\left(z^{\prime}\right) d z^{\prime}},
$$

where $U(z, t ; \mu)$ solves the problem

$$
i \frac{\partial U}{\partial z}+i \dot{\mu} \frac{\partial U}{\partial \mu}-\mu U+\frac{1}{2} \frac{\partial^{2} U}{\partial t^{2}}+|U|^{2} U=-\frac{m}{2} D_{1}(z) \frac{\partial^{2} U}{\partial t^{2}}
$$

with the initial data $U\left(0, t ; \mu_{0}\right)=\Phi\left(t ; \mu_{0}\right)$ and $\mu(0)=\mu_{0}$. The transformation $(2.4)$ describes the adiabatically varying orbit of the NLS soliton (2.3). We present the asymptotic solution of $(2.5)$ as a perturbation series for $U(z, t ; \mu)$ and $\mu(z)$ in powers of $m$ :

$$
U(z, t ; \mu)=\sum_{k=0}^{\infty} m^{k} U^{(k)}(z, t ; \mu)
$$

and

$$
\dot{\mu}=\sum_{k=1}^{\infty} m^{2 k} \Gamma^{(2 k)}(\mu)
$$

where $\Gamma^{(2 k)}(\mu)$ are corrections of the Fermi golden rule for radiative decay of NLS solitons. Substitution of (2.6)-(2.7) into (2.5) produces a chain of equations for corrections of the perturbation series. At the leading, first and second orders, the chain of perturbative equations takes the form

$$
\begin{aligned}
& i \frac{\partial U^{(0)}}{\partial z}-\mu U^{(0)}+\frac{1}{2} \frac{\partial^{2} U^{(0)}}{\partial t^{2}}+\left|U^{(0)}\right|^{2} U^{(0)}=0 \\
& i \frac{\partial U^{(1)}}{\partial z}-\mu U^{(1)}+\frac{1}{2} \frac{\partial^{2} U^{(1)}}{\partial t^{2}}+2\left|U^{(0)}\right|^{2} U^{(1)}+U^{(0) 2} \bar{U}^{(1)}=-\frac{1}{2} D_{1}(z) \frac{\partial^{2} U^{(0)}}{\partial t^{2}}
\end{aligned}
$$

and

$$
\begin{aligned}
& i \frac{\partial U^{(2)}}{\partial z}-\mu U^{(2)}+\frac{1}{2} \frac{\partial^{2} U^{(2)}}{\partial t^{2}}+2\left|U^{(0)}\right|^{2} U^{(2)}+U^{(0) 2} \bar{U}^{(2)} \\
& \quad=-i \Gamma^{(2)}(\mu) \frac{\partial U^{(0)}}{\partial \mu}-\frac{1}{2} D_{1}(z) \frac{\partial^{2} U^{(1)}}{\partial t^{2}}-2\left|U^{(1)}\right|^{2} U^{(0)}-U^{(1) 2} \bar{U}^{(0)} .
\end{aligned}
$$

Initial conditions for these equations are

$$
U^{(0)}\left(0, t ; \mu_{0}\right)=\Phi\left(t ; \mu_{0}\right), \quad \mu(0)=\mu_{0},
$$


and

$$
U^{(k)}\left(0, t ; \mu_{0}\right)=0, \quad k \geq 1 .
$$

Order $\mathrm{O}(1)$. The nonlinear equation (2.8) at order $\mathrm{O}(1)$ with initial data (2.11) has a unique solution, $U^{(0)}(z, t ; \mu)=\Phi(t ; \mu)$, which is the NLS soliton with the adiabatic change of $\mu=\mu(z)$.

Order $\mathrm{O}(m)$. The linear inhomogeneous equation $(2.9)$ at order $\mathrm{O}(m)$ has the Fourier series solution

$$
U^{(1)}(z, t ; \mu)=\sum_{n=-\infty}^{\infty} U_{n}^{(1)}(z, t ; \mu) e^{2 \pi i n z},
$$

where $U_{0}^{(1)}=0$ and $\left(U_{n}^{(1)}, \bar{U}_{-n}^{(1)}\right)$ at $n \geq 1$ solve the coupled equations

$$
\begin{aligned}
& i \frac{\partial U_{n}^{(1)}}{\partial z}-(\mu+2 \pi n) U_{n}^{(1)}+\frac{1}{2} \frac{\partial^{2} U_{n}^{(1)}}{\partial t^{2}}+\Phi^{2}(t ; \mu)\left(2 U_{n}^{(1)}+\bar{U}_{-n}^{(1)}\right) \\
& \quad=-\frac{d_{n}}{2} \Phi^{\prime \prime}(t ; \mu), \\
& -i \frac{\partial \bar{U}_{-n}^{(1)}}{\partial z}-(\mu-2 \pi n) \bar{U}_{-n}^{(1)}+\frac{1}{2} \frac{\partial^{2} \bar{U}_{-n}^{(1)}}{\partial t^{2}}+\Phi^{2}(t ; \mu)\left(2 \bar{U}_{-n}^{(1)}+U_{n}^{(1)}\right) \\
& \quad=-\frac{d_{n}}{2} \Phi^{\prime \prime}(t ; \mu) .
\end{aligned}
$$

It follows from (2.12) that the system (2.14)-(2.15) is supplemented with zero initial conditions: $U_{n}^{(1)}\left(0, t ; \mu_{0}\right)=0$ for any $|n| \geq 1$. Solutions of the system (2.14)-(2.15) are constructed in Appendix A with the use of the spectral decomposition for a linearized NLS operator $[19,20]$. Asymptotic limits of the correction terms $U_{n}^{(1)}(z, t ; \mu)$ are obtained in Appendix B with the use of generalized functions. These calculations show that the continuous-wave radiation in the solution $U_{n}^{(1)}(z, t ; \mu)$ at large distance $z$ and time $t$ is given by the following expression [see (A.1) and (B.9)]:

$$
\lim _{|t| \rightarrow \infty, z \rightarrow \infty} U_{-n}^{(1)}=-\frac{\pi i \sqrt{2 \mu} d_{-n}\left(k_{n}+i\right)^{2}}{4 k_{n}} \operatorname{sech} \frac{\pi k_{n}}{2} e^{i \sqrt{2 \mu} k_{n}|t|}, \quad n \geq N_{\mu},
$$

and

$$
\lim _{|t| \rightarrow \infty, z \rightarrow \infty} U_{-n}^{(1)}=0, \quad n<N_{\mu}
$$

where

$$
k_{n}=\sqrt{\frac{2 \pi n}{\mu}-1}>0, \quad N_{\mu}=\left[\frac{\mu}{2 \pi}\right] .
$$

This result will be used at order $\mathrm{O}\left(m^{2}\right)$ to calculate the decay rate $\Gamma^{(2)}(\mu)$ of DM solitons.

Order $\mathrm{O}\left(\mathrm{m}^{2}\right)$. Solution of the linear inhomogeneous equation (2.11) at order $\mathrm{O}\left(\mathrm{m}^{2}\right)$ can also be represented by the Fourier series:

$$
U^{(2)}(z, t ; \mu)=\sum_{n=-\infty}^{\infty} U_{n}^{(2)}(z, t ; \mu) e^{2 \pi i n z} .
$$


Since the right-hand side of (2.11) has a nonzero mean term in $z$, the nonzero mean term $U_{0}^{(2)}(z, t ; \mu)$ satisfies the inhomogeneous equation

$$
\begin{gathered}
i \frac{\partial U_{0}^{(2)}}{\partial z}-\mu U_{0}^{(2)}+\frac{1}{2} \frac{\partial^{2} U_{0}^{(2)}}{\partial t^{2}}+\Phi^{2}(t ; \mu)\left(2 U_{0}^{(2)}+\bar{U}_{0}^{(2)}\right)=-i \Gamma^{(2)}(\mu) \frac{\partial \Phi(t ; \mu)}{\partial \mu} \\
-\sum_{n=-\infty}^{\infty}\left(\frac{1}{2} d_{-n} \frac{\partial^{2} U_{n}^{(1)}}{\partial t^{2}}+2 \Phi(t ; \mu) U_{n}^{(1)} \bar{U}_{n}^{(1)}+\Phi(t ; \mu) U_{n}^{(1)} U_{-n}^{(1)}\right) .
\end{gathered}
$$

The mean term in the right-hand side of (2.20) leads to a secular growth of $U_{0}^{(2)}(z, t ; \mu)$ in $z$ unless the right-hand side of (2.11) is orthogonalized with respect to eigenfunctions of the kernel of the linearized operator (the Fredholm alternative theorem). The correction $\Gamma^{(2)}(\mu)$ is found from the orthogonalization constraint as follows. Projecting (2.20) onto $\Phi(t ; \mu)$ and subtracting a complex conjugate equation, we obtain a single equation under the condition that $U_{0}^{(2)}(z, t ; \mu)$ is bounded in $t$ :

$$
\begin{aligned}
i \frac{\partial}{\partial z}\left\langle\Phi, U_{0}^{(2)}+\bar{U}_{0}^{(2)}\right\rangle= & -i \Gamma^{(2)}(\mu) \frac{\partial}{\partial \mu}\langle\Phi, \Phi\rangle-\frac{1}{2} \sum_{n=-\infty}^{\infty}\left\langle\Phi^{\prime \prime}, d_{-n} U_{n}^{(1)}-\bar{d}_{-n} \bar{U}_{n}^{(1)}\right\rangle \\
& -\sum_{n=-\infty}^{\infty}\left\langle\Phi^{2}, U_{n}^{(1)} U_{-n}^{(1)}-\bar{U}_{n}^{(1)} \bar{U}_{-n}^{(1)}\right\rangle,
\end{aligned}
$$

where $\langle f, g\rangle$ is the standard inner product in $L^{2}(\mathbb{R})$ :

$$
\langle f, g\rangle=\int_{-\infty}^{\infty} \bar{f}(t) g(t) d t
$$

The right-hand side of (2.21) can be simplified with the use of the system (2.14)-(2.15) as follows:

$$
\begin{aligned}
i \frac{\partial}{\partial z}\left|U_{n}^{(1)}\right|^{2}+\frac{1}{2} \frac{\partial}{\partial t}\left(\bar{U}_{n}^{(1)} \frac{\partial U_{n}^{(1)}}{\partial t}-U_{n}^{(1)} \frac{\partial \bar{U}_{n}^{(1)}}{\partial t}\right)= & -\frac{1}{2} \Phi^{\prime \prime}(t ; \mu)\left(\bar{d}_{-n} \bar{U}_{n}^{(1)}-d_{-n} U_{n}^{(1)}\right) \\
& -\Phi^{2}(t ; \mu)\left(\bar{U}_{n}^{(1)} \bar{U}_{-n}^{(1)}-U_{n}^{(1)} U_{-n}^{(1)}\right) .
\end{aligned}
$$

As a result, the projection formula (2.21) takes the form

$$
\begin{aligned}
& i \frac{\partial}{\partial z}\left[\left\langle\Phi, U_{0}^{(2)}+\bar{U}_{0}^{(2)}\right\rangle+\sum_{n=-\infty}^{\infty}\left\langle U_{n}^{(1)}, U_{n}^{(1)}\right\rangle\right] \\
& \quad=-i \Gamma^{(2)}(\mu) \frac{\partial}{\partial \mu}\langle\Phi, \Phi\rangle-\left.\frac{1}{2} \sum_{n=-\infty}^{\infty}\left(\bar{U}_{n}^{(1)} \frac{\partial U_{n}^{(1)}}{\partial t}-U_{n}^{(1)} \frac{\partial \bar{U}_{n}^{(1)}}{\partial t}\right)\right|_{t=-\infty} ^{t=\infty}
\end{aligned}
$$

It follows from (B.1) and (B.5) of Appendix B for finite $t$ that $\left\langle U_{n}^{(1)}, U_{n}^{(1)}\right\rangle$ becomes $z$-independent in the limit $z \rightarrow \infty$. It also follows from (2.16) that the limiting values of $U_{n}^{(1)}$ at $|t| \gg 1$ are nonzero and constant in the limit $z \rightarrow \infty$ for large negative $n \leq-N_{\mu}$, where $N_{\mu}=\left[\frac{\mu}{2 \pi}\right]$ is the integer ceiling of $\frac{\mu}{2 \pi}>0$. Therefore, we conclude that the correction term $U_{0}^{(2)}(z, t ; \mu)$ is free of secular terms in $z$ in the limit $z \rightarrow \infty$ only if $\Gamma^{(2)}(\mu)$ is defined by the nonlinear Fermi golden rule,

$$
\Gamma^{(2)}(\mu)=-\left.\frac{\sqrt{2 \mu}}{4 i} \sum_{n=-\infty}^{-N_{\mu}} \lim _{z \rightarrow \infty}\left(\bar{U}_{n}^{(1)} \frac{\partial U_{n}^{(1)}}{\partial t}-U_{n}^{(1)} \frac{\partial \bar{U}_{n}^{(1)}}{\partial t}\right)\right|_{t=-\infty} ^{t=\infty},
$$


where we use the formula

$$
\frac{\partial}{\partial \mu}\langle\Phi, \Phi\rangle=\frac{\sqrt{2}}{\sqrt{\mu}}
$$

Using (2.16), we transform (2.24) to the explicit form

$$
\Gamma^{(2)}(\mu)=-\frac{\pi^{2} \mu^{2}}{4} \sum_{n=N_{\mu}}^{\infty} \frac{\left|d_{n}\right|^{2}\left(1+k_{n}^{2}\right)^{2}}{k_{n}} \operatorname{sech}^{2}\left(\frac{\pi k_{n}}{2}\right) .
$$

Assuming $\lim _{n \rightarrow \infty}\left|d_{n}\right|^{2}=0$, the infinite series in (2.26) converges when $\mu \neq \mu_{n} \equiv$ $2 \pi n$, where $n$ is any positive integer. Critical resonances occur at $\mu=\mu_{n}$, when $k_{n}=0$. This case will be studied in more detail in section 3 .

The correction term $U_{0}^{(2)}(z, t ; \mu)$ solves the linear inhomogeneous equation (2.20) under the constraint (2.26). The right-hand side of (2.20) is bounded but nondecaying in the limits $|t| \rightarrow \infty$ and $z \rightarrow \infty$ because of the asymptotic limit (2.16). The nondecaying terms in (2.16) are not in resonance with the left-hand side of (2.20) since $k_{n}^{2}+1=\frac{2 \pi n}{\mu} \neq 0$ for $n \neq 0$. As a result, we conclude from (2.20) that a solution $U_{0}^{(2)}(z, t ; \mu)$ exists and is bounded in the limit $z \rightarrow \infty$ under the condition (2.26). Similarly, one can show that a bounded solution exists for any $U_{n}^{(2)}(z, t ; \mu)$ where $n$ is an integer; i.e., the bounded right-hand side term $D_{1}(z) U_{t t}^{(1)}$ in $(2.11)$ is not in resonance with the left-hand side of (2.11). This completes consideration of the order $\mathrm{O}\left(\mathrm{m}^{2}\right)$ of the perturbation series expansions.

3. Decay rates of DM solitons. Formula (2.26) generalizes the Fermi golden rule for radiative decay of bound states in a linear Schrödinger equation with timeperiodic potentials $[17,18]$. The correction term $\Gamma^{(2)}(\mu)$ is always negative, such that the dynamical system (2.7) exhibits a simple behavior of a monotonic decay of $\mu(z)$ to zero, starting with any initial value $\mu(0)=\mu_{0}>0$. Therefore, the DM soliton decays due to parametric resonances and radiative losses. The decay rate of $\mu(z)$ depends on the nonlinear function $\Gamma^{(2)}(\mu)$ in $(2.26)$. Here we study solutions of the truncated equations (2.7) and (2.26) at the order of $\mathrm{O}\left(\mathrm{m}^{2}\right)$ :

$$
\frac{d \mu}{d z}=-m^{2} \pi^{4} \sum_{n=N_{\mu}}^{\infty} \frac{\left|d_{n}\right|^{2} n^{2}}{k_{n}} \operatorname{sech}^{2}\left(\frac{\pi k_{n}}{2}\right) .
$$

We choose the dispersion coefficient $D_{1}(z)$ as a two-step symmetric function,

$$
D_{1}(z)= \begin{cases}1, & \bmod (z, 1) \in\left(0, \frac{1}{4}\right) \cup\left(\frac{3}{4}, 1\right), \\ -1, & \bmod (z, 1) \in\left(\frac{1}{4}, \frac{3}{4}\right) .\end{cases}
$$

For this dispersion map, the DM soliton is chirp-free at $\bmod (z, 1)=0$ and $\bmod (z, 1)=$ $\frac{1}{2}$ (see [21], for instance). The Fourier coefficients $d_{n}$ for this dispersion map are

$$
d_{n}=\frac{2(-1)^{n+1}}{\pi n} \sin \left(\frac{\pi n}{2}\right) .
$$

As a result, the dynamical system (3.1) takes an explicit form,

$$
\frac{d \mu}{d z}=-4 \pi^{2} m^{2} \sum_{\substack{n=N_{\mu} \\ n \text { odd }}}^{\infty} \frac{1}{k_{n}} \operatorname{sech}^{2}\left(\frac{\pi k_{n}}{2}\right), \quad k_{n}=\sqrt{\frac{2 \pi n}{\mu}-1} .
$$


This equation is the main result of this paper. It describes the radiation damping of DM solitons in the normalized dispersion-periodic NLS equation (2.2) with piecewiseconstant dispersion maps. It is asymptotically accurate when $m \ll 1$ and $\mu$ is not close to critical values $\mu_{n}=2 \pi n$, where $n$ is a positive odd integer. If $\mu \approx \mu_{n}$, critical resonances occur and radiation tails become large, such that the perturbation series breaks down in a strict mathematical sense. The decay-rate function $\Gamma^{(2)}(\mu)$ in the right-hand side of (3.4) for $m=1$ is plotted in Figure 1.

A similar equation for the radiative decay of DM solitons in the presence of weak sinusoidal dispersion variation has been derived in [13]. In that paper, only one term appears in the right-hand side of (3.1) since the Fourier series for $D_{\epsilon}(z)$ in (1.4) contains only a single term in that case.

Below, we analyze the dynamical equation (3.4) under three different limits: (i) $\mu \ll 1$; (ii) $\mu=\mathrm{O}(1$ ); (iii) $\mu \gg 1$.

1. Limit of small values of $\mu$. When $\mu \ll 1$, all terms in the series in (3.4) are present since $N_{\mu}=1$. But only the first term with $n=1$ dominates, since the higher terms are exponentially smaller in $\mu$ compared to the (exponentially small) first term. Therefore, the dynamical equation (3.4) can be truncated at the first term and simplified as

$$
\frac{d \mu}{d z}=-\frac{16 \pi^{2} m^{2} e^{-\pi k_{1}}}{k_{1}}, \quad k_{1}=\sqrt{\frac{2 \pi}{\mu}-1} .
$$

Comparison between numerical solutions of the simplified equation (3.5) and the original equation (3.4) indicates that the simplified equation (3.5) gives a very good approximation to the original equation (3.4) not only for $\mu \ll 1$, but also for $\mu<2 \pi$ (see Figures 2 and 3).

In the limit $\mu \rightarrow 0$, methods of exponential asymptotics can be developed after further simplification of the dynamical equation (3.5):

$$
\frac{d \mu}{d z}=-\alpha m^{2} \mu^{1 / 2} \exp \left(-\frac{\beta}{\mu^{1 / 2}}\right),
$$

where $\alpha=4(2 \pi)^{3 / 2}$ and $\beta=\pi(2 \pi)^{1 / 2}$. In this limit, the radiation damping of DM solitons and the continuous-tail radiation emitted by the DM soliton are exponentially weak. This agrees with the asymptotic beyond-all-orders calculations by Yang and Kath [10]. A similar situation occurs in the dynamics of embedded solitons in the perturbed integrable fifth-order KdV equation in the small velocity limit [22].

Results of [10] are valid when $\mu \ll 1$ and $m$ is arbitrary, while our results are valid when $m \ll 1$ and $\mu$ arbitrary. In the regime of common validity, i.e., $m \ll 1$ and $\mu \ll 1$, the two results match each other, as shown next. When $\mu \ll 1$, the radiation field is dominated by the $n=-1$ term in the Fourier-series solution (2.13) for $U^{(1)}(z, t ; \mu)$. The amplitude of this radiation field is thus given asymptotically from $(2.16)$ as

$$
u_{\mathrm{rad}}=2 m \pi^{\frac{1}{2}} \exp \left(-\frac{\pi^{3 / 2}}{\sqrt{2 \mu}}\right) .
$$

Due to the rescaling of variables (2.1) and different notations, results of [10] need to be reformulated. In the present notations, the amplitude of the radiation field obtained in $[10]$ is in the form

$$
u_{\mathrm{rad}}=\frac{1}{2} C \pi^{\frac{1}{2}} \exp \left(-\frac{\pi^{3 / 2}}{\sqrt{2 \mu}}\right)
$$




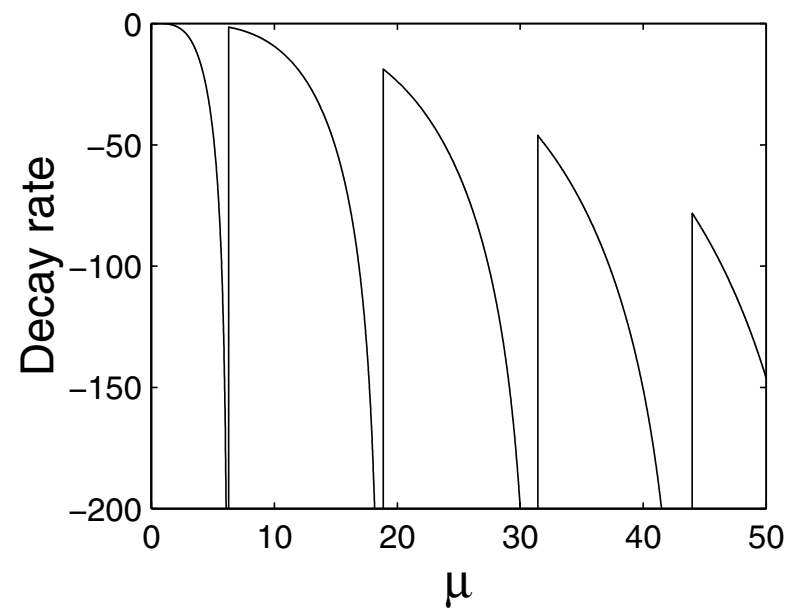

FIG. 1. Decay rate $\Gamma^{(2)}(\mu)$ of DM solitons versus the parameter $\mu$ as in (3.4) for $m=1$.

where $C$ is a dispersion map-dependent constant given in Figure 1 of [10]. Our parameter $m$ is equal to $\frac{1}{2}\left(\sigma_{2}-\sigma_{1}\right)$ of [10]. When $m \ll 1$, inspection of Figure 1 in [10] shows that $C \approx 2\left(\sigma_{2}-\sigma_{1}\right)=4 m$. Thus, in the limits $m, \mu \ll 1$, the radiation field (3.7) from our analysis agrees perfectly with (3.8) from [10]. We note that Yang and Kath [10] also found windows of low radiation field at large values of $m$. Since our results are valid only in the limit $m \ll 1$ and up to the order of $\mathrm{O}\left(m^{2}\right)$, the lowradiation windows cannot be recovered in our analysis unless the perturbation series (2.6) and (2.7) are extended to at least $\mathrm{O}\left(\mathrm{m}^{4}\right)$.

The dynamical equation (3.6) can be integrated with the help of the Laplace method as follows:

$$
\frac{1}{2} \alpha m^{2}\left(z+z_{0}\right)=\exp \left(\frac{\beta}{\mu^{1 / 2}}\right)\left[\frac{\mu}{\beta}+\mathrm{O}\left(\mu^{3 / 2}\right)\right],
$$

where $z_{0}$ is a constant of integration. The leading-order asymptotic solution for $\mu(z)$ in the limit $\mu \rightarrow 0$ is derived from (3.9) in the form

$$
\mu(z)=\left[\frac{\beta}{\log \left[\frac{\alpha m^{2}}{2 \beta}\left(z+z_{0}\right) \log ^{2}\left(z+z_{0}\right)\right]+\mathrm{O}\left(\frac{1}{\log \left(z+z_{0}\right)}\right)}\right]^{2} .
$$

As $z \rightarrow \infty$, the parameter $\mu(z)$ decays logarithmically as

$$
\mu(z) \sim \frac{\beta^{2}}{\log ^{2} z}\left[1-4 \frac{\log \log z}{\log z}\right] .
$$

This logarithmic decay of bound states has been reported previously for internal modes of envelope solitons in [23]. Logarithmic decay is associated with an exponentially small Fermi golden rule for exponentially small radiative waves.

2. Solutions near critical values $\mu_{n}$. If $\mu(0)>2 \pi$, the decay of DM solitons always leads to the point where the parameter $\mu$ has to pass through a critical value $\mu_{n}=2 \pi n$, where $n$ is a positive odd integer. When this happens, the radiation field becomes large, and the perturbation-series solution formally breaks down. Consequently, the 
solution of the dynamical equation (3.4) may no longer give a good approximation to the true solution. However, numerical results indicate that the solution of (3.4) still agrees qualitatively with the solution of the full equation (2.2) (see Figure 4). Here we derive the solution of the dynamical equation (3.4) when it passes through a single critical value $\mu=\mu_{N}$ at $z=z_{N}$.

Since $N_{\mu}$ is an integer ceiling of $\frac{\mu}{2 \pi}$, and $N_{\mu}$ is odd, the one-sided limit $z \rightarrow z_{N}^{-}$is nonsingular, and the parameter $\mu(z)$ approaches $\mu_{N}$ with a linear slope:

$$
\mu(z)=\mu_{N}-\mu_{N}^{\prime}\left(z-z_{N}\right)+\mathrm{O}\left(z-z_{N}\right)^{2}, \quad z<z_{N},
$$

where

$$
\mu_{N}^{\prime}=4 m^{2} \pi^{2} \sum_{\substack{n=N_{\mu} \\ n \text { odd }}}^{\infty} \frac{1}{k_{n}} \operatorname{sech}^{2}\left(\frac{\pi k_{n}}{2}\right),
$$

and $k_{n}$ are all computed at $\mu=\mu_{N}$. Once the parameter $\mu(z)$ passes below $\mu_{N}$, a singular term with $n=N$ appears in the dynamical equation (3.4) because $k_{N}=0$ at $\mu=\mu_{N}$. The leading-order asymptotic approximation for the solution $\mu(z)$ for $z>z_{N}$ takes the form

$$
\mu(z)=\mu_{N}-\left[\alpha\left(z-z_{N}\right)\right]^{2 / 3}+\mathrm{O}\left(z-z_{N}\right), \quad z>z_{N},
$$

where $\alpha=6 m^{2} \pi^{2} \sqrt{\mu_{N}}$. The slope of $\mu(z)$ is infinite in the limit $z \rightarrow z_{N}^{+}$, but the solution $\mu(z)$ is still continuous at $z=z_{N}$. The asymptotic solution (3.14) describes a sharp drop in the amplitude of the DM soliton after it passes through a critical resonance value $\mu_{N}$.

3. Limit of large values of $\mu$. When $\mu \gg 1$, the dynamical equation (3.4) can also be simplified. Using the formula

$$
k_{n+2}^{2}-k_{n}^{2}=\frac{4 \pi}{\mu}
$$

and the Riemann sum approximation for the integral with areas of rectangles, we approximate the sum as

$$
\begin{aligned}
\frac{1}{\mu} \sum_{\substack{n=N_{\mu} \\
n \text { odd }}}^{\infty} \frac{1}{k_{n}} \operatorname{sech}^{2}\left(\frac{\pi k_{n}}{2}\right) & =\frac{1}{2 \pi} \sum_{\substack{n=N_{\mu} \\
n \text { odd }}}^{\infty} \operatorname{sech}^{2}\left(\frac{\pi k_{n}}{2}\right) \frac{\left(k_{n+2}+k_{n}\right)}{2 k_{n}}\left(k_{n+2}-k_{n}\right) \\
& \approx \frac{1}{2 \pi} \int_{k_{0}(\mu)}^{\infty} \operatorname{sech}^{2}\left(\frac{\pi k}{2}\right) d k,
\end{aligned}
$$

where $k_{0}(\mu)=k_{N_{\mu}}$ such that $0<k_{0}(\mu)<1$. In this approximation, the dynamical system (3.4) simplifies to the form

$$
\frac{d \mu}{d z}=-4 m^{2} \mu\left[1-\tanh \left(k_{0}(\mu)\right)\right] .
$$

Using the comparison principle for (3.17), we conclude that DM solitons decay with a linear decay rate when $\mu(z) \gg 1$ :

$$
\mu(0) \exp \left(-4 m^{2} z\right) \leq \mu(z) \leq \mu(0) \exp \left(-4 m^{2} \alpha_{0} z\right),
$$


where $\alpha_{0}=1-\tanh 1>0$.

We note that when $\mu(0) \gg 1$, the monotonic decay of $\mu(z)$ passes through many critical values, where radiation amplitudes are large. As a result, the asymptotic solution (3.18) may not give a good quantitative approximation to the true solution. Nevertheless, the solution (3.18) still describes qualitatively the decay of DM solitons for $\mu(0) \gg 1$ (see Figure 5).

4. Numerical simulations of DM solitons. Here we directly simulate the normalized dispersion-periodic NLS equation (2.2) and compare numerical solutions with the above analytical solutions. Our numerical method uses the fast Fourier transform (FFT) to compute the derivatives in $t$, and the fourth-order Runge-Kutta scheme to advance in $z$. At the values of $z$ where the dispersion has a discontinuity (i.e., $\bmod (z, 1)=\frac{1}{4}$ and $\left.\bmod (z, 1)=\frac{3}{4}\right)$, the stepsize $\Delta z$ is reduced so that the overall fourth-order accuracy in $z$ is assured. To eliminate radiation reflection at the boundaries of the $t$-interval, damping boundary conditions are used. Our results are checked with longer $t$-intervals, more grid points in $t$, and smaller stepsize $\Delta z$, and the results are found to remain the same.

Our numerical simulation starts with the initial condition of a standard (unchirped) NLS soliton:

$$
u(0, t)=\sqrt{2 \mu_{0}} \operatorname{sech} \sqrt{2 \mu_{0}} t .
$$

It is known that DM solitons are unchirped in the middle point of each constantdispersion segment, i.e., at $\bmod (z, 1)=0$ and $\bmod (z, 1)=1 / 2$ in the present case. Thus, when the unchirped NLS soliton (4.1) is launched at $z=0$, the radiation emission is minimal compared to that of chirped solitons.

Below, we describe numerical computations with $m=0.1$ and four different values of $\mu(0)$.

1. Figure 2: $\mu(0)=1$. Figure 2(a) shows the soliton amplitude versus distance $z$. We see that this soliton's amplitude is oscillating (breathing) with unit period, which is the period of the dispersion map $D_{1}(z)$. This behavior is a signature of DM solitons. The evolution of the average soliton amplitude in $z$ is plotted in Figure 2(b). This average amplitude is numerically calculated for each unit distance $z$ as the average between the maximum and minimum amplitudes. It is clear from Figure 2(b) that the DM soliton slowly decays due to the parametric resonance between the soliton and the dispersion map, in accordance with the analytical prediction above. Also in Figure 2(b), the analytical values of the average soliton amplitude $\sqrt{2 \mu}$ obtained from the dynamical equation (3.4) and its simplified version (3.5) are plotted as circles "o" and crosses "x," respectively. We see that both analytical equations (3.4) and (3.5) agree with numerical values and with each other extremely well. This comparison confirms that the dynamical equation (3.4) for radiation damping of DM solitons is asymptotically accurate in the case $m \ll 1$ and $\mu(0)<\mu_{1}=2 \pi$, and that the simplified equation (3.5) is a very good approximation to (3.4) not only for $\mu \ll 1$, but also for $\mu=\mathrm{O}(1)$. The soliton profile at $z=2000$ is shown in Figure 2(c) in a logarithmic scale. We clearly see the central DM-pulse is flanked by continuous-wave radiation. The radiation amplitude is nearly constant. This is because the radiation is excited mainly by the lowest-order resonance with $n=1$ in (3.4), and the radiation field is dominated by the lowest-order radiative waves with $n=1$ in (2.16). At $z=2000$, the parameter $\mu$ can be inferred from Figure 2 (b) as roughly 0.6731 , and the radiation field should be dominated by waves 
(a)

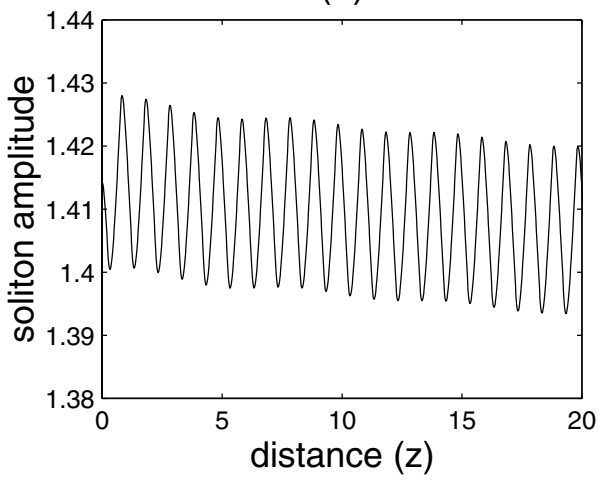

(c)

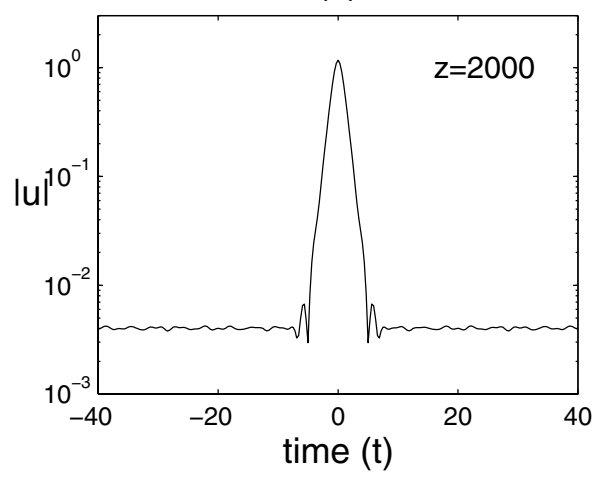

(b)

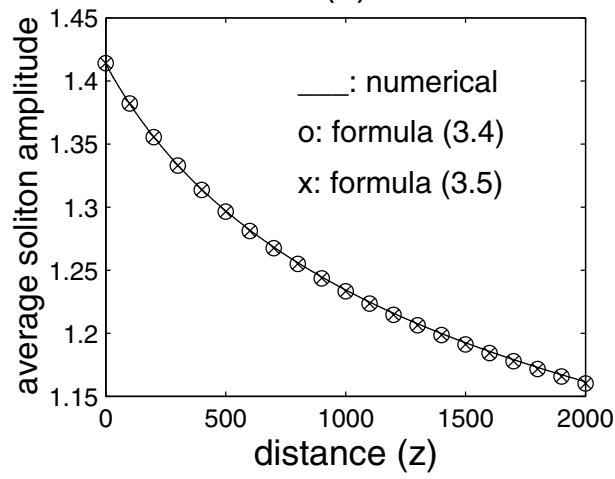

(d)

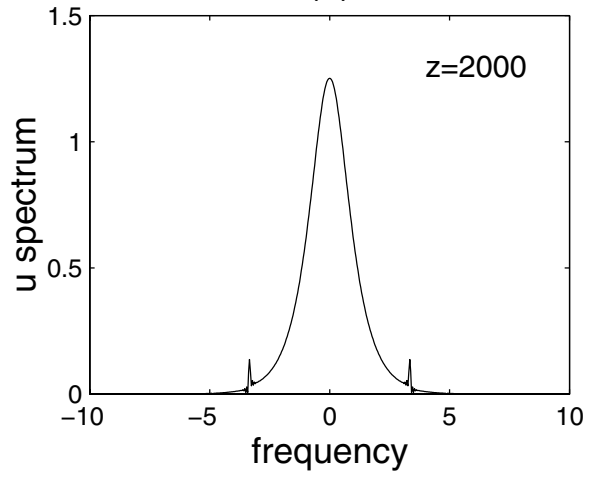

FIG. 2. Numerical evolution of the DM soliton with $m=0.1$ and $\mu(0)=1$. (a) Soliton amplitude versus distance $z$. (b) Average soliton amplitude versus $z$ : numerical results (solid curve); analytical average soliton amplitude $\sqrt{2 \mu}$ from (3.4) (circles); analytical average soliton amplitude $\sqrt{2 \mu}$ from (3.5) (crosses). (c) Solution profile at $z=2000$. (d) Fourier spectrum of the solution at $z=2000$.

with frequencies $\pm \sqrt{2 \mu} k_{1} \approx \pm 3.35$, according to (2.16). This is confirmed in Figure $2(\mathrm{~d})$, where the solution spectrum at $z=2000$ is shown. This spectrum has two spikes at frequencies \pm 3.33 , which are due to the radiation field. The locations of these frequency spikes are in excellent agreement with the theoretical values \pm 3.35 .

2. Figure 3: $\mu(0)=6$. In this case, the initial value of $\mu$ is close to but still below the lowest critical resonance value $\mu_{1}=2 \pi$. Therefore, we expect that the radiation field would be larger, and the theoretical approximation (3.4) for the DM soliton less accurate. This is indeed the case. In Figure 3(a), the soliton amplitude versus distance $z$ is plotted. We see that the amplitude oscillates irregularly, and the period of oscillations is not equal to the unit dispersion map period any-more. This is an indication that the central pulse has deviated from the DM soliton. However, our analytical solution for the average soliton amplitude $\sqrt{2 \mu}$, which is calculated from the dynamical equation (3.4), still gives a very reasonable approximation to the true solution (see the dashed line in Figure 3(a)). We have also compared solutions from the dynamical equation (3.4) and its simplified form (3.5) for the present set of parameters, and found that the two solutions differ by only less than $6 \%$. Thus, over a wide range of $\mu$ values below the critical resonance $\mu_{1}=2 \pi$, the simplified equation (3.5) gives a very good approximation to the original dynamical equation 
(a)

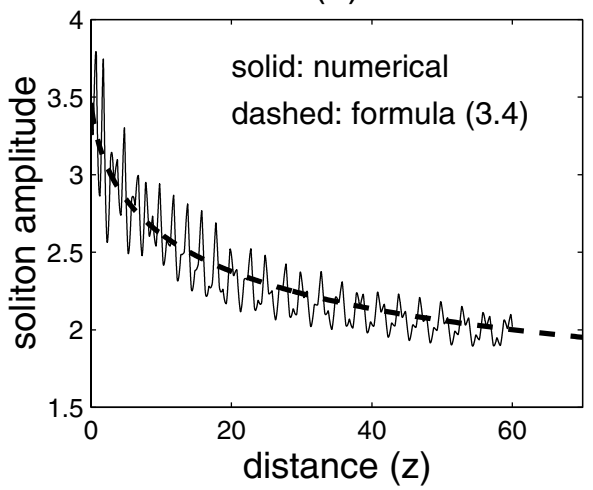

(b)

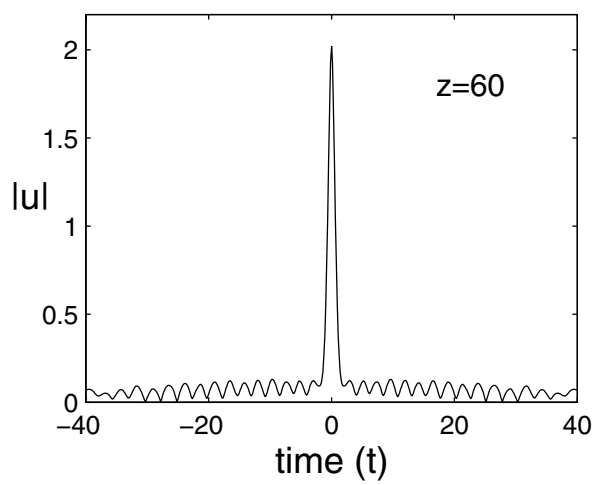

(c)

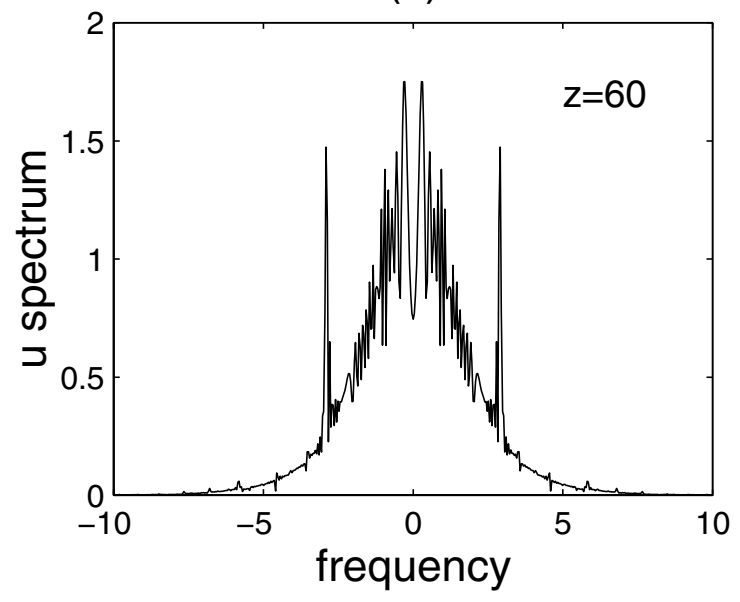

FIG. 3. Numerical evolution of the DM soliton with $m=0.1$ and $\mu(0)=6$. (a) Soliton amplitude versus $z$ : numerical results (solid curve); analytical average soliton amplitude $\sqrt{2 \mu}$ from (3.4) (dashed curve). (b) Solution profile at $z=60$. (c) Spectrum of the solution at $z=60$.

(3.4). In Figures 3(b) and (c), the numerically obtained field profile and its Fourier spectrum at distance $z=60$ are plotted. Due to the quasi-critical resonance, the radiation field in Figure 3(b) is much larger than that in Figure 2(c). In addition, the Fourier spectrum in Figure 3(c) indicates that the solution can no longer be called a DM soliton. Nevertheless, the main resonant spikes on the two sides of Figure 3(c) are still well predicted by the resonance conditions at $k= \pm \sqrt{2 \mu} k_{1}$.

3. Figure 4: $\mu(0)=12$. In this case, the initial value of $\mu$ is above the lowest critical resonance value $\mu_{1}=2 \pi$, and the monotonic decay of the DM soliton passes through this critical resonance. Here we focus on how this transition occurs. In Figure 4(a), the soliton amplitude versus distance $z$ is plotted as the solid curve. We see that radiation damping is initially slow, as the average soliton amplitude decreases toward the critical value at $\sqrt{2 \mu_{1}} \approx 3.54$. In this process, the DM soliton oscillates with the unit period of the dispersion map. A solution profile plotted in Figure 4(b) at $z=50$ shows a weak radiation field, which is the reason for the slow decay of the DM soliton. The corresponding Fourier spectrum in Figure 4(d) shows that the radiation field consists of a discrete set of frequencies which are precisely the resonant frequencies. 
When the average soliton amplitude passes through $\sqrt{2 \mu_{1}}$, a critical resonance occurs. Consequently, the soliton decays much faster (see Figure 4(a)). Strong continuous-wave radiation is emitted in this process, and the DM soliton is strongly modified. After the average soliton amplitude passes below $\sqrt{2 \mu_{1}}$, the pulse oscillates irregularly, and its oscillation period is no longer equal to the unit period of the dispersion map. A solution profile shown in Figure 4(c) at $z=100$ confirms that the radiation field becomes much stronger past the critical-resonance stage. The Fourier spectrum in Figure 4(e) shows that the radiation field is no longer dominated by a discrete set of resonant frequencies. In addition, the Fourier spectrum appears to be quite noisy.

When a critical resonance is reached, the perturbation-series solution (2.6) and (2.7) formally breaks down, and the analytical results are not expected to provide quantitatively accurate approximations to the numerical solution. This is indeed the case. In Figure 4(a), the analytical average soliton amplitude $\sqrt{2 \mu}$ obtained from (3.4) is also plotted (dashed line). We see that prior to the critical resonance, the analytical curve closely follows the numerical average soliton amplitude (not shown). However, when the numerical solution gets close to the critical resonance, it starts to deviate from the analytical curve considerably. In fact, the numerical solution passes through the critical resonance much earlier than what the theory predicts (see Figure 4(a)). Nevertheless, the analytical solution still agrees qualitatively with the numerical solution. For instance, the sharp (infinite-slope) drop of the soliton amplitude as predicted in (3.14) does occur past the critical value of the soliton amplitude at $\sqrt{2 \mu_{1}} \approx 3.54$ (see Figure $4(\mathrm{a})$ ).

4. Figure 5: $\mu(0)=100$. When the initial value of $\mu$ is large, the asymptotic analysis predicts that the soliton decays exponentially according to the bounds in (3.18). However, the monotonic soliton-decay passes through many critical resonances in this case. Thus, the accuracy of the analytical prediction needs to be examined. To address this issue, the results from numerical simulations at $m=0.1$ and $\mu(0)=100$ are shown in Figures $5(\mathrm{a})-(\mathrm{e})$. When $\mu \gg 1$, the DM soliton spends most of the time inside individual constant-dispersion segments, where the DM soliton is governed by the standard NLS equation. This is reflected by the fast amplitude oscillations inside each constant-dispersion segment in Figure 5(a). Due to the radiative damping, the DM soliton passes through critical resonances at $n=15,13,11,9,7, \ldots, 1$, when the average soliton amplitude matches the critical values at $\sqrt{4 \pi n}=13.73,12.78,11.76,10.63,9.38, \ldots, 3.54$, respectively. It follows from Figure 5(a) that, even though the DM soliton passes through a number of critical resonances here, it still holds up and maintains its DM soliton character and the unit periodicity up to the first four critical resonances. The solution profile and the Fourier spectrum of the DM soliton at $z=15$ are shown in Figures 5(b) and (d). Further evolution of the DM soliton shows that the DM soliton character is lost after the fifth critical resonance at the average amplitude about 9.38. The solution profile and its Fourier spectrum at $z=24$ are shown in Figures 5(c) and (e). A noisy spectrum past the critical resonances similar to that of Figure 4(e) is observed.

It follows from Figure 5(a) that higher-order critical resonances have a much weaker effect on the dynamics of the DM soliton than do lower-order resonances. As a result, the analytical dashed curve in Figure 5(a) for the average soliton amplitude agrees well with the numerical results until the fifth critical resonance is reached. We have also checked that the analytical curve in Figure 5(a) is indeed bounded between the two exponentially decaying functions in (3.18). 
(a)

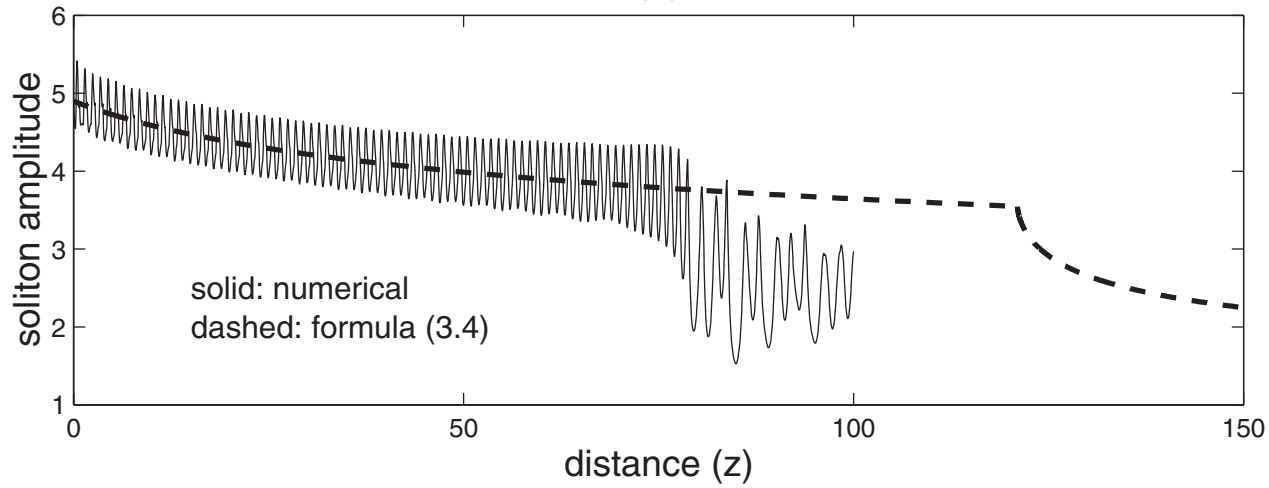

(b)

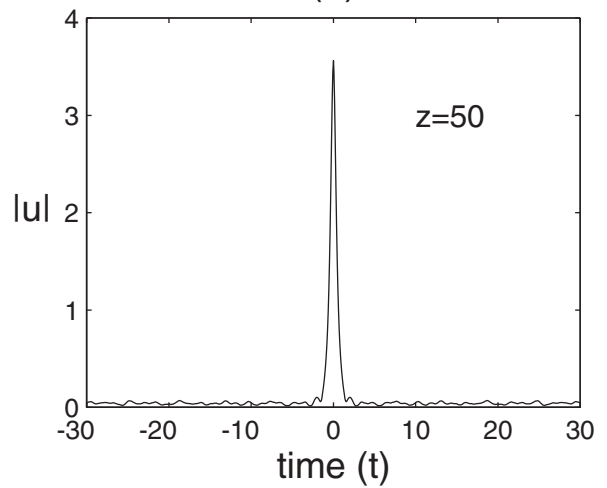

(d)

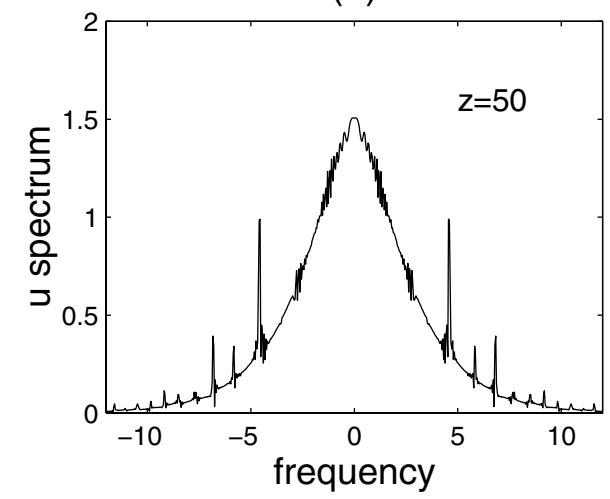

(c)

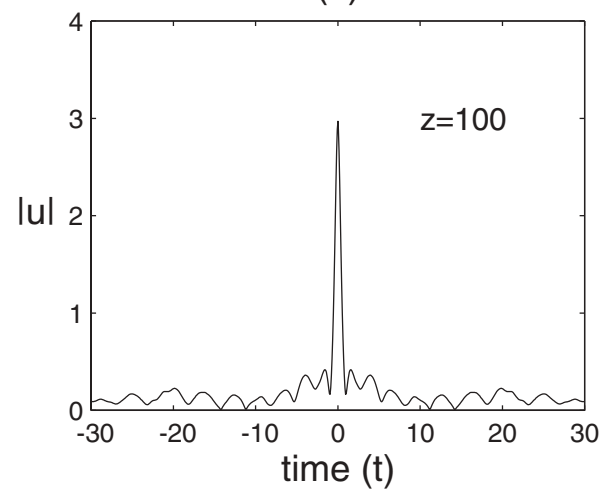

(e)

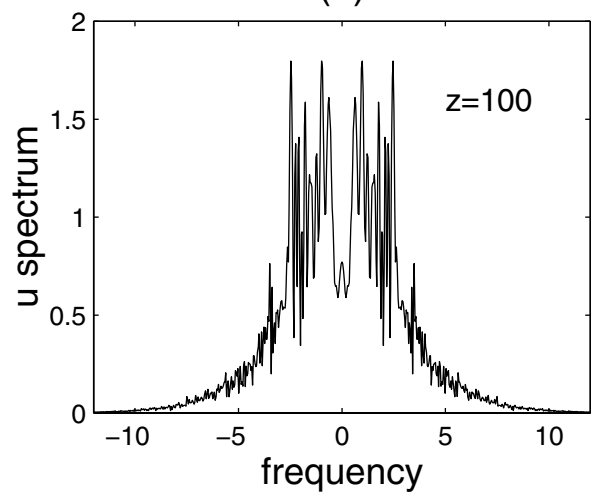

FIG. 4. Numerical evolution of the DM soliton with $m=0.1$ and $\mu(0)=12$. (a) Soliton amplitude versus $z$ : numerical results (solid curve); analytical average soliton amplitude $\sqrt{2 \mu}$ from (3.4) (dashed curve). (b), (c) Solution profiles at $z=50$ and $z=100$. (d), (e) Spectra of the solutions at $z=50$ and $z=100$. 
(a)

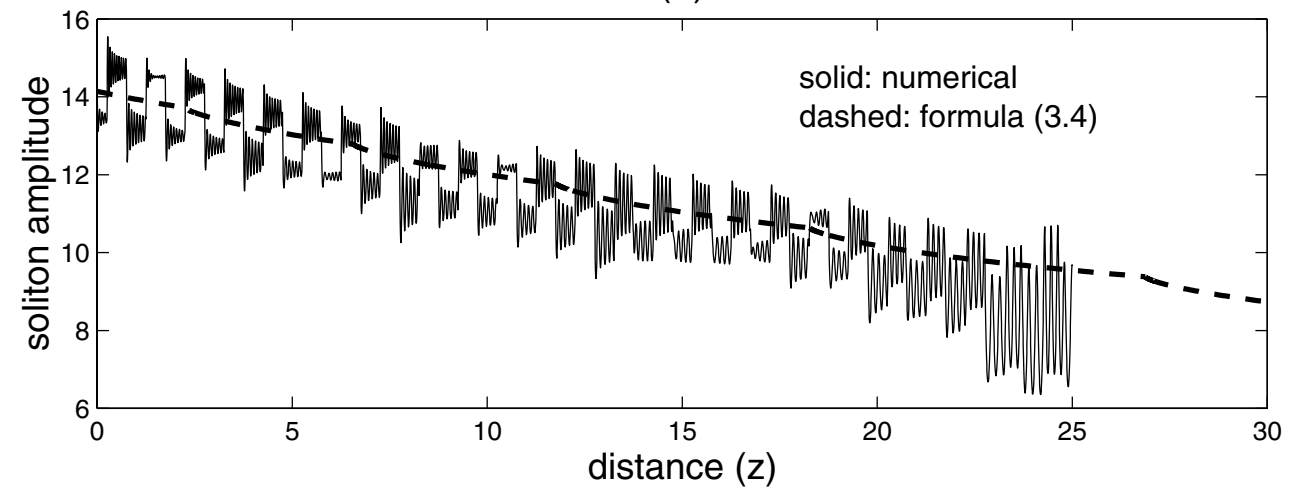

(b)

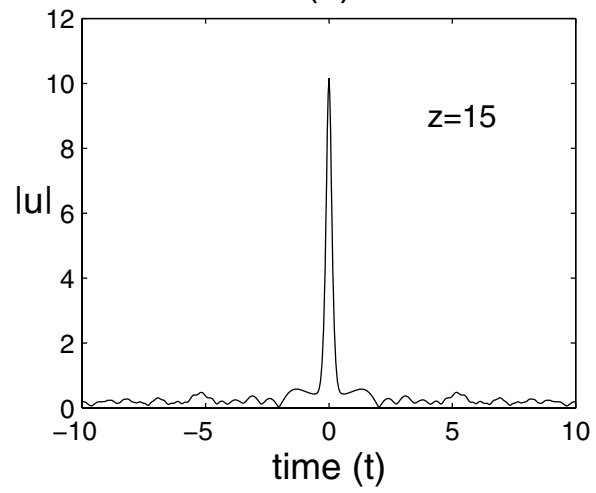

(d)

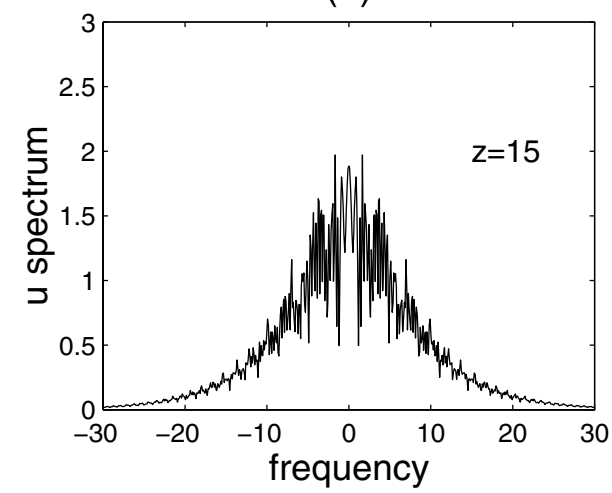

(c)

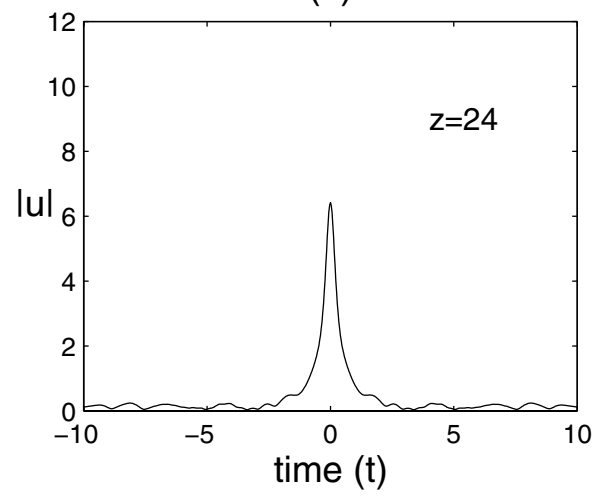

(e)

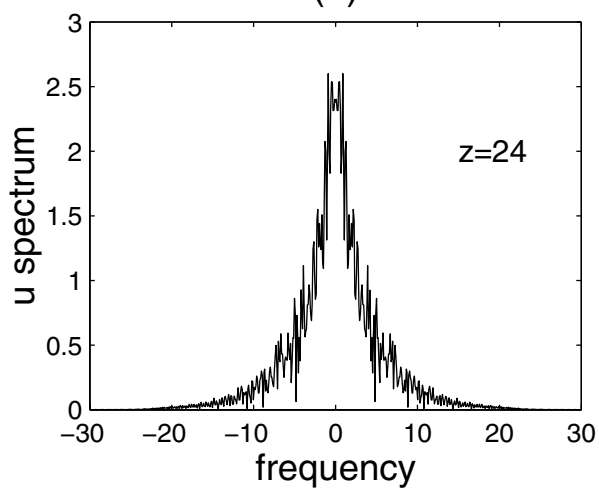

FIG. 5. Numerical evolution of the DM soliton with $m=0.1$ and $\mu(0)=100$. (a) Soliton amplitude versus $z$ : numerical results (solid curve); analytical average soliton amplitude $\sqrt{2 \mu}$ from (3.4) (dashed curve). (b), (c) Solution profiles at $z=15$ and $z=24$. (d), (e) Spectra of the solutions at $z=15$ and $z=24$. 
(a)

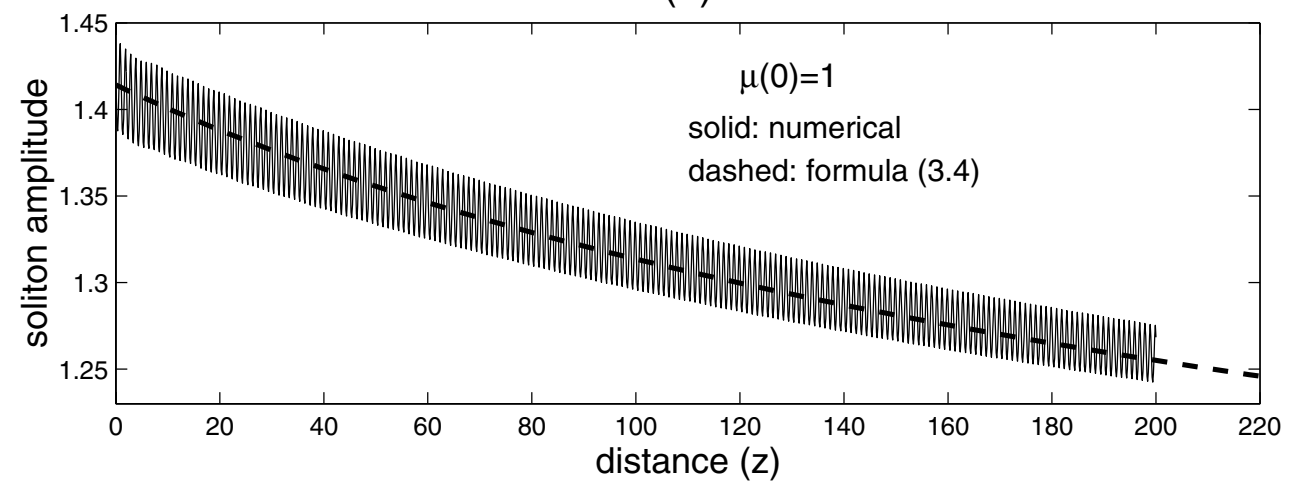

(b)

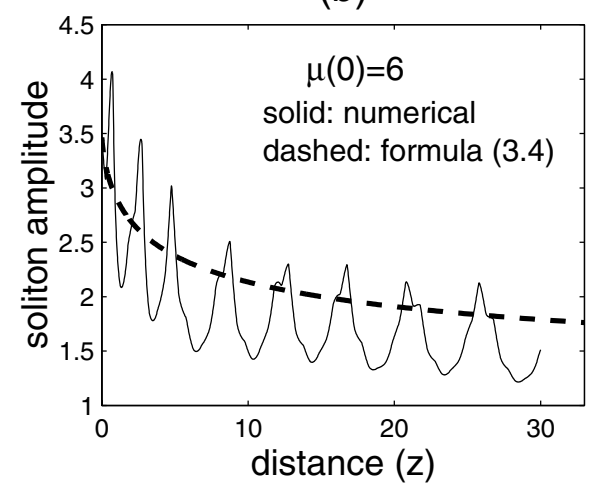

(c)

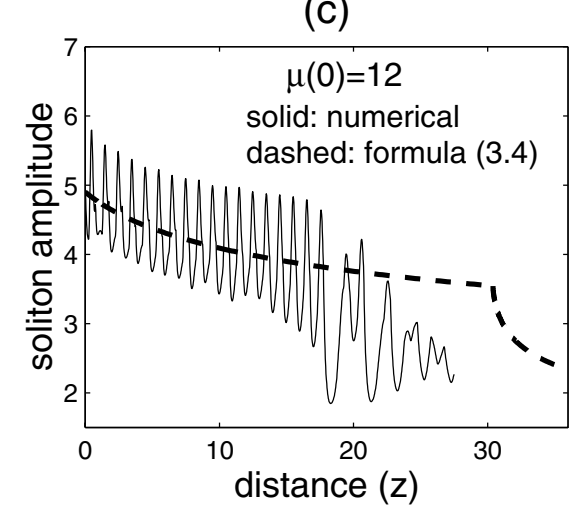

FIG. 6. Numerical evolutions of the DM soliton amplitudes with $m=0.2$ and (a) $\mu(0)=1$; (b) $\mu(0)=6 ;$ (c) $\mu(0)=12$. Numerical results are shown by solid curves. Analytical average soliton amplitudes $\sqrt{2 \mu}$ from (3.4) are shown by dashed curves.

In the end of this section, we discuss how the solution changes when the perturbation strength $m$ gets larger. For this purpose, we choose $m=0.2$, compared to $m=0.1$ in Figures 2-5. The soliton amplitudes versus distance $z$ for $\mu(0)=1,6,12$ are shown in Figures 6(a), (b), and (c), respectively. The average soliton amplitudes predicted from (3.4) are also plotted for comparison. Figure 6(a) shows that in the case of small and moderate values of $\mu(0)$, the soliton still decays according to the analytical equation (3.4). Figures 6(b) and (c) indicate that when $\mu(0)$ is close to or above the lowest critical resonance value $\mu_{1}=2 \pi$, the pulse deviates further from the DM soliton than in the case of $m=0.1$, and the pulse amplitude oscillates with a period further away from the unit period of the dispersion map. When $m$ increases, the distance scale for soliton evolution shrinks by a factor of $\mathrm{m}^{2}$, as formula (3.4) predicts. For instance, when $m=0.1$ and $\mu(0)=12$, the lowest critical resonance is reached in the numerical solution at $z \approx 76$ (see Figure $4(\mathrm{a})$ ), while when $m=0.2$, the critical resonance in the numerical solution is reached at $z \approx 18$, i.e., four times faster.

5. Summary and discussion. In this paper, we have studied the nonlinear parametric resonance of DM solitons for average-anomalous dispersion $\left(D_{0}>0\right)$ in the limit $m \rightarrow 0$ by both analytical and numerical methods. We have found that due to a resonance between the DM soliton and the dispersion map, the soliton 
keeps shedding continuous-wave radiation and consequently decays. The radiation amplitude is on the order of $m$, while the decay rate of DM solitons is on the order of $m^{2}$. We have calculated the analytical approximations for the decay rate of DM solitons in the limits of small, intermediate, and large initial soliton amplitudes. We have shown that when the soliton passes through a critical resonance, it decays much faster. All these analytical results are found to be in excellent agreement with direct numerical simulations.

Resonances in the dispersion-periodic NLS equation (1.1) resemble a nonlinear generalization of parametric resonances in a linear Schrödinger equation studied recently in $[17,18]$. The perturbation term in $[17,18]$ satisfies the assumption of being periodic in time and decaying fast in space. The nonlinear problem (1.1) does not satisfy this localization assumption. In addition, the periodic variations of $D_{\epsilon}(z)$ are not generally small perturbations of the mean term $D_{0}$ in real communication systems. Thus, rigorous analysis of the parametric resonance of DM solitons in dispersionperiodic NLS equation (1.1) with nonsmall dispersion variations needs further investigation.

Appendix A: Solutions of the first-order problem (2.14)-(2.15). We use Kaup's method [19] to solve the inhomogeneous problem (2.14)-(2.15) with the spectral decomposition for a linearized NLS operator. Since the potential of the problem can be rescaled as $\Phi(t ; \mu)=\sqrt{2 \mu} \Phi(T)$, where $\Phi(T)=\operatorname{sech} T$ and $T=\sqrt{2 \mu} t$, we transform the variables as follows:

$$
U_{n}^{(1)}(z, t ; \mu)=2 d_{n} \sqrt{2 \mu} V_{n}(Z, T), \quad Z=\mu z, \quad T=\sqrt{2 \mu} t .
$$

The system (2.14)-(2.15) in new variables transforms to the following:

$$
\begin{gathered}
i \frac{\partial V_{n}}{\partial Z}-\left(1+\lambda_{n}\right) V_{n}+\frac{\partial^{2} V_{n}}{\partial T^{2}}+2 \operatorname{sech}^{2} T\left(2 V_{n}+\bar{V}_{-n}\right)=-\frac{1}{2} \Phi^{\prime \prime}(T) \\
-i \frac{\partial \bar{V}_{-n}}{\partial Z}-\left(1-\lambda_{n}\right) \bar{V}_{-n}+\frac{\partial^{2} \bar{V}_{-n}}{\partial T^{2}}+2 \operatorname{sech}^{2} T\left(2 \bar{V}_{-n}+V_{n}\right)=-\frac{1}{2} \Phi^{\prime \prime}(T)
\end{gathered}
$$

where

$$
\lambda_{n}=\frac{2 \pi n}{\mu} .
$$

The system is written in matrix notations as

$$
\mathcal{L}\left[\begin{array}{c}
V_{n} \\
\bar{V}_{-n}
\end{array}\right]=\left(i \frac{\partial}{\partial Z}-\lambda_{n}\right)\left[\begin{array}{c}
V_{n} \\
\bar{V}_{-n}
\end{array}\right]+\frac{1}{2}\left[\begin{array}{c}
1 \\
-1
\end{array}\right] \Phi^{\prime \prime}(T)
$$

where the linearized NLS operator is

$$
\mathcal{L}=\left[\begin{array}{cc}
-\frac{\partial^{2}}{\partial T^{2}}+1-4 \operatorname{sech}^{2} T & -2 \operatorname{sech}^{2} T \\
2 \operatorname{sech}^{2} T & \frac{\partial^{2}}{\partial T^{2}}-1+4 \operatorname{sech}^{2} T
\end{array}\right] .
$$

The linearized NLS operator $\mathcal{L}$ possesses a complete set of eigenfunctions [19] that consists of eigenfunctions associated with two branches of the continuous spectrum and eigenfunctions associated with the zero eigenvalue of the discrete spectrum. The continuous spectrum eigenfunctions are

$$
\boldsymbol{\psi}_{1}(T ; k)=e^{i k T}\left[\left(1-\frac{2 i k e^{-T}}{(k+i)^{2} \cosh T}\right)\left(\begin{array}{l}
0 \\
1
\end{array}\right)+\frac{1}{(k+i)^{2} \cosh ^{2} T}\left(\begin{array}{l}
1 \\
1
\end{array}\right)\right]
$$


and

$$
\boldsymbol{\psi}_{2}(T ; k)=e^{-i k T}\left[\left(1+\frac{2 i k e^{-T}}{(k-i)^{2} \cosh T}\right)\left(\begin{array}{l}
1 \\
0
\end{array}\right)+\frac{1}{(k-i)^{2} \cosh ^{2} T}\left(\begin{array}{l}
1 \\
1
\end{array}\right)\right]
$$

such that $\mathcal{L} \boldsymbol{\psi}_{1}(T ; k)=-\left(1+k^{2}\right) \boldsymbol{\psi}_{1}(T ; k)$ and $\mathcal{L} \boldsymbol{\psi}_{2}(T ; k)=\left(1+k^{2}\right) \boldsymbol{\psi}_{2}(T ; k)$. The zero eigenvalue has algebraic multiplicity four and geometric multiplicity two. The eigenfunctions of the zero eigenvalue are

$$
\phi_{1}(T)=\left(\begin{array}{c}
1 \\
-1
\end{array}\right) \operatorname{sech} T, \quad \phi_{2}(T)=\left(\begin{array}{l}
1 \\
1
\end{array}\right) \operatorname{sech} T \tanh T,
$$

such that $\mathcal{L} \phi_{1,2}(T)=0$. The generalized eigenfunctions of the zero eigenvalue are

$$
\phi_{1}^{d}(T)=\left(\begin{array}{l}
1 \\
1
\end{array}\right)(T \tanh T-1) \operatorname{sech} T, \quad \phi_{2}^{d}(T)=\left(\begin{array}{c}
1 \\
-1
\end{array}\right) T \operatorname{sech} T,
$$

such that $\mathcal{L} \phi_{1,2}^{d}(T)=2 \phi_{1,2}(T)$. Eigenfunctions of the linearized NLS operator $\mathcal{L}$ satisfy the orthogonality conditions

$$
\begin{gathered}
\left\langle\boldsymbol{\psi}_{1}\left(k^{\prime}\right)\left|\sigma_{3}\right| \boldsymbol{\psi}_{1}(k)\right\rangle=-2 \pi \delta\left(k^{\prime}-k\right), \quad\left\langle\boldsymbol{\psi}_{2}\left(k^{\prime}\right)\left|\sigma_{3}\right| \boldsymbol{\psi}_{2}(k)\right\rangle=2 \pi \delta\left(k^{\prime}-k\right), \\
\left\langle\boldsymbol{\phi}_{1}\left|\sigma_{3}\right| \boldsymbol{\phi}_{1}^{d}\right\rangle=-2, \quad\left\langle\boldsymbol{\phi}_{2}\left|\sigma_{3}\right| \boldsymbol{\phi}_{2}^{d}\right\rangle=2,
\end{gathered}
$$

with respect to the inner product

$$
\left\langle\mathbf{f}\left|\sigma_{3}\right| \mathbf{g}\right\rangle=\int_{-\infty}^{\infty}\left[\bar{f}_{1}(T) g_{1}(T)-\bar{f}_{2}(T) g_{2}(T)\right] d T .
$$

All other inner products computed with eigenfunctions (A.6)-(A.9) are identically zero. The orthogonality conditions (A.10)-(A.11) are modified compared with the original definition in [19]. Orthogonality conditions similar to (A.10)-(A.11) were used by Kaup and Lakoba [20].

The right-hand side term of (A.4) can be decomposed through a complete set of eigenfunctions (A.6)-(A.9) as follows:

$$
\begin{aligned}
\mathbf{F}=\frac{1}{2}\left[\begin{array}{c}
1 \\
-1
\end{array}\right] \Phi^{\prime \prime}(T)= & \int_{-\infty}^{\infty}\left[\alpha(k) \boldsymbol{\psi}_{1}(T ; k)+\beta(k) \boldsymbol{\psi}_{2}(T ; k)\right] d k \\
& +a \phi_{1}(T)+b \boldsymbol{\phi}_{2}(T)+c \boldsymbol{\phi}_{1}^{d}(T)+d \boldsymbol{\phi}_{2}^{d}(T),
\end{aligned}
$$

where the expansion coefficients can be explicitly computed as

$$
\begin{gathered}
\alpha(k)=-\frac{1}{2 \pi}\left\langle\boldsymbol{\psi}_{1}(k)\left|\sigma_{3}\right| \mathbf{F}\right\rangle=\frac{(k+i)^{2}}{8} \operatorname{sech} \frac{\pi k}{2}, \\
\beta(k)=\frac{1}{2 \pi}\left\langle\boldsymbol{\psi}_{2}(k)\left|\sigma_{3}\right| \mathbf{F}\right\rangle=-\frac{(k-i)^{2}}{8} \operatorname{sech} \frac{\pi k}{2}, \\
a=-\frac{1}{2}\left\langle\boldsymbol{\phi}_{1}^{d}\left|\sigma_{3}\right| \mathbf{F}\right\rangle=-\frac{1}{2}, \quad b=\frac{1}{2}\left\langle\phi_{2}^{d}\left|\sigma_{3}\right| \mathbf{F}\right\rangle=0,
\end{gathered}
$$




$$
c=-\frac{1}{2}\left\langle\phi_{1}\left|\sigma_{3}\right| \mathbf{F}\right\rangle=0, \quad d=\frac{1}{2}\left\langle\phi_{2}\left|\sigma_{3}\right| \mathbf{F}\right\rangle=0 .
$$

Here we have used the exact value,

$$
\frac{1}{\pi} \int_{-\infty}^{\infty} \frac{\cos k T}{\cosh T} d T=\operatorname{sech}\left(\frac{\pi k}{2}\right)
$$

The solution of (A.2)-(A.3) can be found by using the spectral decomposition:

$$
\begin{aligned}
{\left[\begin{array}{c}
V_{n} \\
\bar{V}_{-n}
\end{array}\right](Z, T)=} & \int_{-\infty}^{\infty}\left[\alpha_{n}(k, Z) \boldsymbol{\psi}_{1}(T ; k)+\beta_{n}(k, Z) \boldsymbol{\psi}_{2}(T ; k)\right] d k \\
& +a_{n}(Z) \phi_{1}(T)+b_{n}(Z) \phi_{2}(T)+c_{n}(Z) \phi_{1}^{d}(T)+d_{n}(Z) \phi_{2}^{d}(T)
\end{aligned}
$$

Coefficients of the expansion satisfy a simple $Z$-evolution problem with zero initial conditions:

$$
\begin{gathered}
i \frac{\partial \alpha_{n}}{\partial Z}=\left(\lambda_{n}-1-k^{2}\right) \alpha_{n}-\alpha(k), \quad i \frac{\partial \beta_{n}}{\partial Z}=\left(\lambda_{n}+1+k^{2}\right) \beta_{n}-\beta(k), \\
i \frac{\partial a_{n}}{\partial Z}=\lambda_{n} a_{n}+2 c_{n}-a, \quad i \frac{\partial b_{n}}{\partial Z}=\lambda_{n} b_{n}+2 d_{n}-b,
\end{gathered}
$$

and

$$
i \frac{\partial c_{n}}{\partial Z}=\lambda_{n} c_{n}-c, \quad i \frac{\partial d_{n}}{\partial Z}=\lambda_{n} d_{n}-d
$$

The unique solution of the $Z$-evolution problem (A.19)-(A.21) is

$$
\begin{gathered}
\alpha_{n}(k, Z)=\frac{\alpha(k)}{\lambda_{n}-1-k^{2}}\left[1-e^{-i\left(\lambda_{n}-1-k^{2}\right) Z}\right], \\
\beta_{n}(k, Z)=\frac{\beta(k)}{\lambda_{n}+1+k^{2}}\left[1-e^{-i\left(\lambda_{n}+1+k^{2}\right) Z}\right], \\
a_{n}(Z)=-\frac{1}{2 \lambda_{n}}\left[1-e^{-i \lambda_{n} Z}\right], \quad b_{n}(Z)=0,
\end{gathered}
$$

and

$$
c_{n}(Z)=0, \quad d_{n}(Z)=0 .
$$

Equations (A.24)-(A.25) are obtained with the use of (A.16)-(A.17).

Appendix B: Asymptotic limits for the first-order solution. We analyze the first-order solution $\left(V_{n}, \bar{V}_{-n}\right)(Z, T)$ defined in the spectral representation form (A.18) of Appendix A with explicit spectral coefficients in (A.14)-(A.15) and (A.22)(A.25). The asymptotic limit $Z \rightarrow \infty$ depends on a range of values of $T$.

(i) $|T|<\infty$ and $Z \rightarrow \infty$. The first-order solution is a sum of two terms, $V_{n}(Z, T)=W_{n}(T)+Q_{n}(Z, T)$, where $W_{n}(T)$ is generated by the inhomogeneous part of the system (A.2)-(A.3) and $Q_{n}(Z, T)$ is generated by the homogeneous part of the 
system (A.2)-(A.3) in the initial-value problem. Using the spectral decomposition (A.18), we express $W_{n}(T)$ and $Q_{n}(Z, T)$ explicitly as

$$
\left[\begin{array}{c}
W_{n} \\
\bar{W}_{-n}
\end{array}\right](T)=\int_{-\infty}^{\infty}\left[\frac{\alpha(k)}{\lambda_{n}-1-k^{2}} \boldsymbol{\psi}_{1}(T ; k)+\frac{\beta(k)}{\lambda_{n}+1+k^{2}} \boldsymbol{\psi}_{2}(T ; k)\right] d k-\frac{1}{2 \lambda_{n}} \boldsymbol{\phi}_{1}(T)
$$

and

$$
\begin{aligned}
{\left[\begin{array}{c}
Q_{n} \\
\bar{Q}_{-n}
\end{array}\right](Z, T)=} & -\int_{-\infty}^{\infty}\left[\frac{\alpha(k) e^{-i\left(\lambda_{n}-1-k^{2}\right) Z}}{\lambda_{n}-1-k^{2}} \boldsymbol{\psi}_{1}(T ; k)\right. \\
& \left.+\frac{\beta(k) e^{-i\left(\lambda_{n}+1+k^{2}\right) Z}}{\lambda_{n}+1+k^{2}} \boldsymbol{\psi}_{2}(T ; k)\right] d k+\frac{e^{-i \lambda_{n} Z}}{2 \lambda_{n}} \phi_{1}(T) .
\end{aligned}
$$

We use formulas of generalized functions,

$$
\lim _{Z \rightarrow \infty} \frac{e^{ \pm i K Z}}{K}= \pm \pi i \delta(K)
$$

and

$$
\delta\left(k^{2}+k_{n}^{2}\right)=0, \quad \delta\left(k^{2}-k_{n}^{2}\right)=\frac{1}{2 k_{n}}\left[\delta\left(k-k_{n}\right)+\delta\left(k+k_{n}\right)\right],
$$

and notice that the limit $Z \rightarrow \infty$ in (B.2) is nonzero only if the resonance equation $1+k^{2} \pm \lambda_{n}=0$ has a solution for real $k$. We consider $n>0$ such that $\lambda_{n}>0$ and denote $k_{n}=\sqrt{\lambda_{n}-1} \geq 0$ for $\lambda_{n} \geq 1$. The resonance condition $\lambda_{n} \geq 1$ is satisfied for $n \geq N_{\mu}$, where $N_{\mu}=\left[\frac{\mu}{2 \pi}\right]$ is the integer ceiling of $\frac{\mu}{2 \pi}>0$. With the use of (B.3)-(B.4), we compute the limit $Z \rightarrow \infty$ for $Q_{n}(Z, T)$ at $n \geq N_{\mu}$ and finite $T$ :

$$
\lim _{Z \rightarrow \infty}\left[\begin{array}{c}
Q_{n} \\
\bar{Q}_{-n}
\end{array}\right](Z, T)=\frac{\pi i}{2 k_{n}}\left[\alpha\left(k_{n}\right) \boldsymbol{\psi}_{1}\left(T ; k_{n}\right)+\alpha\left(-k_{n}\right) \boldsymbol{\psi}_{1}\left(T ;-k_{n}\right)\right] .
$$

The first-order solution $V_{n}(Z, T)=W_{n}(T)+Q_{n}(Z, T)$ is bounded in $T$ and $Z$ in the limit $Z \rightarrow \infty$.

(ii) $|T| \rightarrow \infty$ and $Z \rightarrow \infty$. It follows from (B.3)-(B.4) that

$$
\lim _{T \rightarrow \pm \infty} \frac{e^{i k T}}{\left(k-k_{n}\right)\left(k+k_{n}\right)}= \pm \frac{\pi i}{2 k_{n}}\left[\delta\left(k-k_{n}\right) e^{i k_{n} T}-\delta\left(k+k_{n}\right) e^{-i k_{n} T}\right] .
$$

Using this formula for $n \geq N_{\mu}$, we find from (B.1) and (B.5) that

$$
\lim _{T \rightarrow \pm \infty}\left[\begin{array}{c}
W_{n} \\
\bar{W}_{-n}
\end{array}\right](T)=\mp \frac{\pi i}{16 k_{n}} \operatorname{sech} \frac{\pi k_{n}}{2}\left[e^{i k_{n} T}\left(k_{n} \pm i\right)^{2}-e^{-i k_{n} T}\left(k_{n} \mp i\right)^{2}\right]\left(\begin{array}{c}
0 \\
1
\end{array}\right)
$$

and

$$
\lim _{T \rightarrow \pm \infty, Z \rightarrow \infty}\left[\begin{array}{c}
Q_{n} \\
\bar{Q}_{-n}
\end{array}\right](Z, T)=\frac{\pi i}{16 k_{n}} \operatorname{sech} \frac{\pi k_{n}}{2}\left[e^{i k_{n} T}\left(k_{n} \pm i\right)^{2}+e^{-i k_{n} T}\left(k_{n} \mp i\right)^{2}\right]\left(\begin{array}{c}
0 \\
1
\end{array}\right) .
$$


As a result, the boundary values of the first-order solution $V_{n}(Z, T)$ satisfy the Sommerfeld radiation boundary conditions:

$$
\lim _{|T| \rightarrow \infty, Z \rightarrow \infty} V_{-n}(Z, T)=-\frac{\pi i\left(k_{n}+i\right)^{2}}{8 k_{n}} \operatorname{sech} \frac{\pi k_{n}}{2} e^{i k_{n}|T|}, \quad n \geq N_{\mu} .
$$

(iii) $|T| \rightarrow \infty$ and $Z<\infty$. Using formula (B.6) in (B.1)-(B.2), we find that both terms cancel out since

$$
\lim _{k \rightarrow \pm k_{n}}\left(1-e^{-i\left(\lambda_{n}-1-k^{2}\right) Z}\right)=0 .
$$

As a result, we have zero boundary values for $V_{n}(Z, T)$ in the limit $|T| \rightarrow \infty$ for finite $Z$ :

$$
\lim _{|T| \rightarrow \infty} V_{n}(Z, T)=0 .
$$

The first-order solution represents radiative waves diverging from the NLS soliton. In the limit $Z \rightarrow \infty$, the radiative waves approach the $Z$-independent boundary values given by (B.9). In the intermediate region, where $|T| \rightarrow \infty, Z \rightarrow \infty$, and $\lim _{Z \rightarrow \infty} T / Z=C$, where $0<C<\infty$, the radiative waves move with the group velocity $2 k_{n}$, according to the intermediate asymptotic expression

$$
\lim _{|T| \rightarrow \infty, Z \rightarrow \infty} V_{-n}(Z, T)=-\frac{\pi i\left(k_{n}+i\right)^{2}}{8 k_{n}} \operatorname{sech} \frac{\pi k_{n}}{2} e^{i k_{n}|T|} H\left(2 k_{n}-\frac{|T|}{Z}\right), \quad n \geq N_{\mu},
$$

where $H(z)=1$ for $z>0$ and $H(z)=0$ for $z<0$. The intermediate asymptotic expression includes (B.9) and (B.10) as particular cases.

Acknowledgments. The authors thank W. Kath, E. Kirr, B. Malomed, M. Weinstein, and V. Zharnitsky for useful discussions.

\section{REFERENCES}

[1] V. Cautaerts, A. Maruta, and Y. Kodama, On the dispersion-managed soliton, Chaos, 10 (2000), p. 515.

[2] S. Turitsyn, M. P. Fedoruk, E. G. Shapiro, V. K. Mezentsev, and E. G. Turitsyna, Novel approaches to numerical modeling of periodic dispersion-managed fiber communication systems, IEEE J. Quantum Electr., 6 (2000), pp. 263-275.

[3] J. H. Nijhof, W. Forysiak, and N. J. Doran, The averaging method for finding exactly periodic dispersion-managed solitons, IEEE J. Quantum Electr., 6 (2000), p. 330.

[4] D. E. Pelinovsky and V. Zharnitsky, Averaging of dispersion-managed solitons: Existence and stability, SIAM J. Appl. Math., 63 (2003), pp. 745-776.

[5] I. R. Gabitov and S. K. TURItsyn, Averaged pulse dynamics in a cascaded transmission system with passive dispersion compensation, Opt. Lett., 21 (1996), pp. 327-329.

[6] M. J. Ablowitz and G. Biondini, Multiscale pulse dynamics in communication systems with strong dispersion management, Opt. Lett., 23 (1998), pp. 1668-1670.

[7] V. Zharnitsky, E. Grenier, S. Turitsyn, C. K. R. T. Jones, and J. S. Hesthaven, Ground states of dispersion-managed nonlinear Schrödinger equation, Phys. Rev. E (3), 62 (2000), p. 7358 .

[8] M. Kunze, The singular perturbation limit of a variational problem from nonlinear fiber optics, Phys. D, 180 (2003), pp. 108-114.

[9] P. M. Lushnikov, Dispersion-managed soliton in a strong dispersion map limit, Opt. Lett., 26 (2001), pp. 1535-1537.

[10] T. YANG AND W. L. Kath, Radiation loss of dispersion-managed solitons in optical fibers, Phys. D, 149 (2001), p. 80. 
[11] J. H. B. Nijhof, N. J. Doran, W. Forysiak, And F. M. Knox, Stable soliton-like propagation in dispersion-managed systems with net anomalous, zero and normal dispersion, Electron. Lett., 33 (1997), pp. 1726-1727.

[12] A. V. Mikhailov and V. Yu. Novokshenov, The Riemann-Hilbert Problem for Analytic Description of the DM Solitons, preprint, University of Leeds, Leeds, UK, 2003.

[13] F. K. Abdullaev, J. G. Caputo, and N. Flytzanis, Envelope soliton propagation in media with temporally modulated dispersion, Phys. Rev. E (3), 50 (1994), pp. 1552-1558.

[14] B. Malomed, D. F. Parker, and N. F. Smyth, Resonant shape oscillations and decay of a soliton in a periodically inhomogeneous nonlinear optical fiber, Phys. Rev. E (3), 48 (1993), p. 1418.

[15] R. Grimshaw, J. He, and B. A. Malomed, Decay of a fundamental soliton in a periodically modulated nonlinear waveguide, Phys. Scripta 53 (1996), pp. 385-393.

[16] F. K. Abdullaev and J. G. Caputo, Validation of the variational approach for chirped pulses in fibers with periodic dispersion, Phys. Rev. E (3), 58 (1998), p. 6637.

[17] P. D. Miller, A. Soffer, And M. I. Weinstein, Metastability of breather modes of timedependent potentials, Nonlinearity, 13 (2000), pp. 507-568.

[18] E. Kirr And M. I. Weinstein, Parametrically excited Hamiltonian partial differential equations, SIAM J. Math. Anal., 33 (2001), pp. 16-52.

[19] D. J. Kaup, Perturbation theory for solitons in optical fibers, Phys. Rev. A (3), 42 (1990), pp. $5689-5694$.

[20] D. J. Kaup and T. I. Lakoba, Variational method: How it can generate false instabilities, J. Math. Phys., 37 (1996), pp. 3442-3462.

[21] T. I. Lakoba, J. Yang, D. J. Kaup, And B. A. Malomed, Conditions for stationary pulse propagation in the strong dispersion management regime, Opt. Comm., 149 (1998), pp. 366-375.

[22] J. YAnG, Dynamics of embedded solitons in the extended KdV equations, Stud. Appl. Math., 106 (2001), p. 337.

[23] D. Pelinovsky, Radiative effects to the adiabatic dynamics of envelope-wave solitons, Phys. D, 119 (1998), pp. 301-313. 